MATHEMATICS OF COMPUTATION

Volume 75, Number 255, July 2006, Pages 1527-1540

S 0025-5718(06)01791-1

Article electronically published on March 28, 2006

\title{
INTEGER TRANSFINITE DIAMETER AND POLYNOMIALS WITH SMALL MAHLER MEASURE
}

\author{
VALÉRIE FLAMMANG, GEORGES RHIN, AND JEAN-MARC SAC-ÉPÉE
}

\begin{abstract}
In this work, we show how suitable generalizations of the integer transfinite diameter of some compact sets in $\mathbb{C}$ give very good bounds for coefficients of polynomials with small Mahler measure. By this way, we give the list of all monic irreducible primitive polynomials of $\mathbb{Z}[X]$ of degree at most 36 with Mahler measure less than 1.324... and of degree 38 and 40 with Mahler measure less than 1.31.
\end{abstract}

\section{INTRODUCTION}

The Mahler measure of a polynomial

$$
P=c_{0} X^{n}+\cdots+c_{n}=c_{0} \prod_{k=1}^{n}\left(X-\alpha_{k}\right) \in \mathbb{C}[X], c_{0} \neq 0,
$$

is

$$
M(P)=\left|c_{0}\right| \prod_{k=1}^{n} \max \left(1,\left|\alpha_{k}\right|\right) .
$$

For an algebraic number $\alpha$, we denote by $M(\alpha)$ the Mahler measure of its minimal polynomial in $\mathbb{Z}[X]$. If $P \in \mathbb{Z}[X]$, we have $M(P) \geq 1$ and the theorem of Kronecker implies that $M(P)=1$ if and only if $P$ is a product of cyclotomic polynomials and a power of $X$. The Lehmer question is the following

Lehmer's question. Does there exist a positive constant $c$ such that, if $M(\alpha)>1$, then $M(\alpha) \geq 1+c$ ?

We say that the polynomial $P$ is reciprocal if $X^{n} P(1 / X)=P(X)$, and that an algebraic number is reciprocal if its minimal polynomial is reciprocal. C.J. Smyth SM71 has proved that, if the algebraic number $\alpha \neq 0,1$ is nonreciprocal, then $M(\alpha) \geq \theta_{0}$, where $\theta_{0}=1.324717 \cdots$ is the smallest Pisot number which is the real root of $X^{3}-X-1$.

P. Voutier $[\mathrm{V}]$ has proved that, if the algebraic number $\alpha$ of degree $n>2$ is not a root of unity, then

$$
M(\alpha) \geq 1+\frac{1}{4}\left(\frac{\log \log n}{\log n}\right)^{3} .
$$

Received by the editor November 24, 2004 and, in revised form, February 8, 2005.

2000 Mathematics Subject Classification. Primary 11Y40, 11R06.

Key words and phrases. Mahler measure, integer transfinite diameter, explicit auxiliary function.

(C)2006 American Mathematical Society Reverts to public domain 28 years from publication 
The smallest value for $M(\alpha)>1$ has been obtained by D.H. Lehmer DHL himself and is $M(\alpha)=1.1762808 \cdots$ given by the polynomial of degree 10 :

$$
L(X)=X^{10}+X^{9}-X^{7}-X^{6}-X^{5}-X^{4}-X^{3}+X+1 .
$$

Exhaustive searches have been made by D.W. Boyd [BO80] for degrees 4 to 16. He wrote "The case $n=18$ is feasible, but potentially expensive, so this has been deferred in the hope of future theoretical progress or else an improved algorithm." Nevertheless he [BO89] and M.J. Mossinghoff [MO and WM] succeeded to complete the search for degree 18 to 20 and 22 to 24 , respectively, to find all the algebraic numbers $\alpha$ with $M(\alpha)<1$. 3. The search for degree 24 involved testing about $9.8 \times 10^{12}$ polynomials. Here we will reduce this number to $1.3 \times 10^{6}$. We give the list $\mathrm{WE}$ of all algebraic integers of degree $\leq 36$ with Mahler measure $<\theta_{0}$ and of degree 38 and 40 with measure $<1.31$ (which is close to Mossinghoff's limit point $1.3090983806 \cdots)$ ) and then prove that Mossinghoff's table WM] is complete up to degree 40.

Other extensive searches have been made by M.J. Mossinghoff [WM] with heuristic methods up to degree 180. G. Rhin and J.-M. Sac-Épée RSE used both a statistical method and a minimization method to get polynomials of large degree and small Mahler measure.

In this paper we give new bounds for the coefficients of the polynomials with small measure. For this, we use a large family of explicit auxiliary functions. These functions are related to a generalization of the integer transfinite diameter.

We follow Boyd's strategy [BO80]. For a fixed $d$ and a fixed $M \leq \theta_{0}$ we put $E_{0}$ as the set of all irreducible polynomials $P$ with $\operatorname{deg}(P)=2 d$ and $M(P)<M$. The first step is to get a large set $E_{1}$ of polynomials $P$ containing $E_{0}$. Then, we eliminate from the set $E_{1}$ a large proportion of polynomials which do not succeed to some tests. We get a new set $E_{2}$. These computations are done with the language Pascal (in double precision). In the last step we use GP-Pari [PARI] to find the final set of desired polynomials $E_{0}$. For this, we use a modified Graeffe algorithm.

In Section 2, we give some notations and a brief scheme of the computations. In Section 3, we recall the method of explicit auxiliary functions as it was used to study the particular case of small Salem numbers. In Section 4, we define the generalized integer transfinite diameter closely related to the previous explicit auxiliary function of Section 3. In Section 5, we explain how we generalize the situation of Sections 3 and 4 to the case of polynomials with small Mahler measure and how Wu's algorithm [WU] and the semi-infinite linear programming method provide good explicit auxiliary functions. This gives bounds for $s_{k}(1 \leq k \leq 40)$, where $s_{k}$ is the sum of the $k$ th powers of the roots of $P$ and then bounds for the coefficients of $P$. In Section 6 we give new bounds for $s_{2 k}$ depending on the values of $s_{k}$. Then we get the set $E_{1}$. In Section 7 we explain how to get the subset $E_{2}$. Section 8 is devoted to the final computations to get $E_{0}$.

\section{The TECHNique FOR THE COMPUTATION}

2a. Notations. Because of Smyth's theorem, an algebraic integer with Mahler measure less than $\theta_{0}$ is reciprocal. Its minimal polynomial $P$ is of degree $2 d$

$$
P=X^{2 d}+c_{1} X^{2 d-1}+\cdots+c_{2 d-1} X+1=\prod_{i=1}^{2 d}\left(X-\alpha_{i}\right) .
$$


We may suppose that $\left|\boldsymbol{\alpha}_{i}\right| \geq 1$ and $\boldsymbol{\alpha}_{d+i}=1 / \boldsymbol{\alpha}_{i}$ for $1 \leq i \leq d$. We define the polynomial $Q$, associated to the polynomial $P$ by the formula $X^{d} Q(X+1 / X)=$ $P(X)$. Therefore

$$
Q=X^{d}+b_{1} X^{d-1}+\cdots+b_{d-1} X+b_{d}
$$

is a monic polynomial of degree $d$ with integer coefficients whose roots are $\gamma_{i}=\boldsymbol{\alpha}_{i}+1 / \boldsymbol{\alpha}_{i}$ for $1 \leq i \leq d$.

We define $s_{1}=\sum_{1 \leq i \leq d} \gamma_{i}=\sum_{1 \leq i \leq 2 d} \boldsymbol{\alpha}_{i}$. More generally for $k>1$ we define $\gamma_{i, k}=\boldsymbol{\alpha}_{i}^{k}+1 / \boldsymbol{\alpha}_{i}^{k}$ and $s_{k}=\sum_{1 \leq i \leq d} \gamma_{i, k}=\sum_{1 \leq i \leq 2 d} \boldsymbol{\alpha}_{i}^{k}$. Let $1<M \leq \theta_{0}$ be a fixed bound. If we suppose that $1 \leq\left|\boldsymbol{\alpha}_{i}\right| \leq M^{a}$ with $0<a \leq 1$, then $\gamma_{i}$ lies inside the ellipse

$$
\mathcal{E}_{a}=\left\{z=x+i y \text { such that }\left(\frac{x}{A}\right)^{2}+\left(\frac{y}{B}\right)^{2} \leq 1\right\},
$$

where $A=M^{a}+M^{-a}$ and $B=M^{a}-M^{-a}$. We denote by $\mathcal{E}_{0}$ the interval $[-2,2]$.

2b. A more general set of polynomials. The bounds for the coefficients of the polynomials $P$ in the set $E_{0}$ are also valid for more general polynomials. Since the polynomials with $M(P)<1.33$ and $2 d<16$ have been already computed in BO80, we suppose now that $2 d \geq 16$. For $M$ fixed, we assume that the polynomials $P$ satisfy the following conditions:

$1 P$ is a monic reciprocal polynomial of degree $2 d$ with integer coefficients, and $M(P)<M$. Its roots are, as before, the numbers $\boldsymbol{\alpha}_{i}$ for $1 \leq i \leq 2 d$ and $Q$, is the polynomial associated to $P$.

Let $k$ be a fixed integer. Let $R_{k}^{+}$(respectively $R_{k}^{-}$) be the polynomial whose roots are the numbers $\boldsymbol{\alpha}_{i}^{k}$ (respectively $-\boldsymbol{\alpha}_{i}^{k}$ ) for $1 \leq i \leq 2 d$. We say that the polynomial $P$ satisfies the property $\mathcal{C}_{k}$ if no factor of the polynomials $Q$ associated to $R_{k}^{+}$or $R_{k}^{-}$divides any of the polynomials $Q_{j}$ which belong to a finite set $S$ which will be defined later.

2 For $1 \leq j \leq 40$, the polynomial $P$ has the property $\mathcal{C}_{j}$.

2c. The technique for computation of the set $E_{1}$. The main task of the calculation is to compute bounds for the quantities $s_{k}$ for $1 \leq k \leq 40$. This is done by using a family of explicit auxiliary functions. For small $k$, this method drastically improves the classical bounds. Then, using Newton's formula, $s_{k} c_{0}+s_{k-1} c_{1}+\cdots+s_{1} c_{k-1}+k c_{k}=0$, we obtain by induction bounds for the coefficients $c_{k}$ for $1 \leq k \leq d$. At this point, all the coefficients of $P$ are known because $P$ is reciprocal. Then we get the set $E_{1}$ of polynomials $P$ (and $Q$ ). There are two cases to examine.

$2 \mathrm{~d}$. The first case. In the first case we suppose that $P$ has a root $\boldsymbol{\alpha}_{1}$ of modulus greater than $M^{1 / 2}$. Since $\boldsymbol{\alpha}_{1}$ is real, we may suppose that $\boldsymbol{\alpha}_{1}$ is positive. We divide this case in 6 subcases: $1 / 2+(j-1) / 12 \leq \log \left|\boldsymbol{\alpha}_{1}\right| / \log M<1 / 2+j / 12$ for $1 \leq j \leq 6$. For each $j$ the numbers $\gamma_{2}, \ldots, \gamma_{d}$ belong to the ellipse $\mathcal{E}_{1 / 2-(j-1) / 12}$, and, therefore, they are close to the real axis. Moreover, we will see that the worst situation occurs when $d-2$ roots $\boldsymbol{\alpha}_{i}$ are of modulus 1, i.e., the corresponding numbers $\gamma_{i, k}$ belong to $\mathcal{E}_{0}$, and one root is of modulus $M^{1 / 2-(j-1) / 12}$. 
2e. The second case. In the second case all the roots $\boldsymbol{\alpha}_{i}$ of $P$ for $1 \leq i \leq d$ are of modulus $\leq M^{1 / 2}$. We will see that, in this case, the worst situation occurs when $d-2 \operatorname{roots} \boldsymbol{\alpha}_{i}$ are of modulus 1 and two are of modulus $M^{1 / 2}$. We may assume that the polynomial $Q$ has the following property: the first nonzero coefficient $b_{i}$ with $i$ odd is positive.

2f. Conclusion of Section 2. Then, in any case and in the worst situation, at least $d-2$ roots of $Q$ are in the interval $(-2,2)$. So, it is of particular interest to study what happens when all the roots of $Q$ are real $>-2$ and only one is $>2$, that is to say, when $P$ is the minimal polynomial of a Salem number. This situation is investigated in the next section.

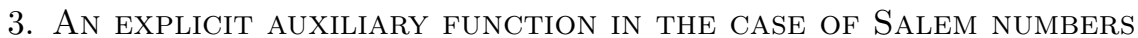

3a. Definition. A Salem number is a positive algebraic integer $\tau>1$ whose one conjugate $(1 / \tau)$ lies inside the open unit disk, and all the others have modulus 1 . The minimal polynomial $P$ of $\tau$ is reciprocal of degree $2 d$. We define the polynomial $Q$ as in Section 2. $Q$ is a polynomial with $d$ real roots, and all but $\gamma_{1}=\tau+1 / \tau>2$ are in the real interval $(-2,2)$.

3b. A lower bound for the trace of Salem numbers. We define the explicit auxiliary function $f$ by the formula

$$
f(x)=x-\sum_{1 \leq j \leq J} e_{j} \log \left|Q_{j}(x)\right|,
$$

where the polynomials $Q_{j}$ are nonzero elements of $\mathbb{Z}[X]$ and the numbers $e_{j}$ are positive real numbers. We denote by $m$ the minimum of the function $f(x)$ for $x>$ -2 and $Q_{j}(x) \neq 0$ for $1 \leq j \leq J$. Such an auxiliary function was given by Smyth SM84 with $m=-0.2281$. It was slightly improved in FGR to $m=-0.2264606$ and recently by Smyth SM03] to $m=-0.2216214$. In this case $J=17$ and for all $j \operatorname{deg} Q_{j} \leq 10$. We have $\sum_{1 \leq i \leq d} f\left(\gamma_{i}\right) \geq d m$ and

$$
s_{1} \geq d m+\sum_{1 \leq j \leq J} e_{j} \log \left|\prod_{1 \leq i \leq d} Q_{j}\left(\gamma_{i}\right)\right| .
$$

We assume now that the polynomial $P$ satisfies the conditions of Section 2 where the set $S$ is the set of polynomials $Q_{j}$. Then $\prod_{1<i<d} Q_{j}\left(\gamma_{i}\right)$ is a nonzero rational integer, because it is the resultant of $Q$ and $Q_{j}$. Therefore

$$
s_{1} \geq d m .
$$

3c. Upper and lower bounds for $s_{k}$ in the case of Salem numbers. For $k>1, \tau^{k}$ is also a Salem number; then we get the same lower bound for $s_{k}$. We now replace $\gamma_{i, k}$ by $-\gamma_{i, k}$ in the previous summation. We now suppose that $\tau<\theta_{0}$. Since $f$ is an increasing function in $(-\infty,-2)$, we get the following inequality:

$$
-s_{k} \geq(d-1) m+f\left(-\theta_{0}^{k}-\theta_{0}^{-k}\right) .
$$


3d. Improvement of the lower bound for $s_{k}$. Now, we want to get good lower bounds for $s_{k}$ when $\tau$ belongs to a small interval, for example to the last interval of the first case of Section 2: $t_{1}=\theta_{0}^{11 / 12}=1.294036 \cdots \leq \tau<\theta_{0}$. The function $f$ is increasing in the interval $[3.03, \infty)$ so, if $t_{1}^{k}+t_{1}^{-k}>3.03$, i.e., for $k>4$, the inequality (3.2) may be replaced by

$$
s_{k} \geq(d-1) m+f\left(t_{1}^{k}+t_{1}^{-k}\right) .
$$

3e. A numerical example for the degree 34. We give an example to show that the bounds for $s_{k}$ are fairly good. The numbers $\tau_{1}=1.296210 \cdots$ and $\tau_{2}=$ $1.324667 \cdots$ are two Salem numbers of degree 34 which belong to the interval $\left[t_{1}, \theta_{0}\right)$. Their minimal polynomials are

$$
\begin{aligned}
P_{1}= & X^{34}-X^{33}-X^{31}+X^{29}+X^{27}-2 X^{26}+X^{23}+X^{22}-X^{21} \\
& -X^{20}-X^{19}+X^{18}+X^{17}+X^{16}-X^{15}-X^{14}-X^{13}+X^{12} \\
& +X^{11}-2 X^{8}+X^{7}+X^{5}-X^{3}-X+1
\end{aligned}
$$

and

$P_{2}=X^{34}-X^{32}-X^{29}-X^{26}-X^{23}-X^{20}-X^{17}-X^{14}-X^{11}-X^{8}-X^{5}-X^{2}+1$,

respectively. The conditions on $P_{1}$ and $P_{2}$ are satisfied because the degrees of the polynomials $Q_{j}$ are at most 10 . We give in Table 1 , for some values of $k$, the lower bound (min) for $s_{k}$ given by (3.2) or (3.4), the values of $s_{k, 1}$ and $s_{k, 2}$, for $P_{1}$ and $P_{2}$ respectively, and the upper bound $(\max )$ for $s_{k}$ given by $(3.3)$. In the last line we give the value of the classical upper bound (MAX) for $\left|s_{k}\right|$, which is $M^{k}+2 d-2+M^{-k}$.

TABLE 1.

\begin{tabular}{|c|c|c|c|c|c|c|c|c|c|c|}
\hline$k$ & 1 & 2 & 3 & 4 & 6 & 8 & 10 & 20 & 30 & 40 \\
\hline $\min$ & -3 & -3 & -3 & -3 & -3 & -1 & 3 & 157 & 2259 & 30021 \\
\hline$s_{k, 1}$ & 1 & 1 & 4 & 5 & 4 & 13 & 11 & 175 & 2399 & 32149 \\
\hline$s_{k, 2}$ & 0 & 2 & 0 & 2 & 2 & 10 & 17 & 277 & 4607 & 76605 \\
\hline $\max$ & 4 & 6 & 7 & 9 & 13 & 18 & 27 & 295 & 4635 & 76758 \\
\hline MAX & 34 & 34 & 34 & 35 & 37 & 41 & 48 & 308 & 4642 & 76757 \\
\hline
\end{tabular}

3f. Conclusion of Section 3. This explicit auxiliary function is sufficient to compute all Salem numbers $\tau$ which are less than a reasonable bound (say 1.3 as in [FGR] and with $\operatorname{deg}(\tau) \leq 40$. We remark that there is no need for the second case because $\tau^{2}$ would belong to the first case. On the one hand, since in the first case of small Mahler measure the nonreal roots of $Q$ are not too far from the real axis, this auxiliary function is certainly not a bad one. On the other hand, in the second case, it is useful to get a better auxiliary function adapted to this particular situation.

Therefore, it is suitable to understand how to construct such functions in more general situations. For this purpose, we first show, in the next section, how these explicit auxiliary functions are connected to a generalization of the integer transfinite diameter. 


\section{Relations BETWEen EXPLiCit AUXiLiARY FUnCTIONS AND INTEGER TRANSFINITE DIAMETER}

4a. A new form for $f(x)$. If, inside the auxiliary function of (3.1), we replace the real numbers $e_{j}$ by rational numbers, we may write

$$
f(x)=x-\frac{t}{h} \log |H(x)|,
$$

where $H$ is in $\mathbb{Z}[X]$ of degree $h$ and $t$ is a positive real number. We want to get a function $f$ whose minimum $m$ on $(-2, \infty)$ is as large as possible. That is to say, we seek a polynomial $H \in \mathbb{Z}[X]$ such that

$$
\sup _{x>-2}|H(x)|^{t / h} e^{-x} \leq e^{-m}
$$

Now, if we suppose that $t$ is fixed, say $t=1$, it is clear that we need to get an effective upper bound for the quantity

$$
t_{\mathbb{Z}, \boldsymbol{\varphi}}([-2, \infty))=\liminf _{\substack{h \geq 1 \\ h \rightarrow \infty}} \inf _{\substack{H \in \mathbb{Z}[X] \\ e \operatorname{deg} H=h}} \sup _{x>-2}|H(x)|^{t / h} \boldsymbol{\varphi}(x)
$$

in which we use the weight $\varphi(x)=e^{-x}$. To get an upper bound for $t_{\mathbb{Z}, \varphi}([-2, \infty))$, it is sufficient to get an explicit polynomial $H \in \mathbb{Z}[X]$ and then to use the sequence of the successive powers of $H$. Let us now define the usual integer transfinite diameter.

4b. The integer transfinite diameter. Let $K$ be a compact subset of $\mathbb{C}$. If $P$ is a polynomial in $\mathbb{C}[X]$ we put $|P|_{\infty, K}=\sup _{z \in K}|P(z)|$. We define the integer transfinite diameter of $K$ by

$$
t_{\mathbb{Z}}(K)=\liminf _{\substack{n \geq 1 \\ n \rightarrow \infty}} \min _{\substack{P \in \mathbb{Z}[X] \\ \operatorname{deg} P=n}}|P|_{\infty, K}^{1 / n} .
$$

For any $n \geq 1$ we say that a polynomial $P_{n}$ (not always unique) is a Chebyshev polynomial if $\left|P_{n}\right|_{\infty, K}=\min _{P \in \mathbb{Z}[X], \operatorname{deg}(P)=n}|P|_{\infty, K}$. It is known that, if $K=[a, b]$ is a real interval of length $b-a \geq 4$, then $t_{\mathbb{Z}}(K)=\frac{b-a}{4}$. However, if $b-a<4$, then $t_{\mathbb{Z}}(K)<1$, but in this case the exact value of $t_{\mathbb{Z}}(K)$ is not known. For more details see [BE], [FRS] or [PR].

4c. How to construct an explicit auxiliary function. It can be seen that in (4.1), if $t$ is fixed, we have a generalization of the classical integer transfinite diameter. Even though we have replaced the set $K$ by an infinite interval, it is clear that, because of the weight $\varphi$, the quantity $t_{\mathbb{Z}, \boldsymbol{\varphi}}([-2, \infty))$ is finite. In general, to get a good upper bound for $t_{\mathbb{Z}}$ (we recall that $t$ is fixed), we need a polynomial $H$ of large degree $h$ (about $10^{8}$ ), but it is not possible to compute a Chebyshev polynomial $P_{h}$ of such a large degree $h$. So we will proceed as follows. For a fixed $t$, it is possible to use Wu's algorithm [WU to compute Chebyshev polynomials, or at least polynomials whose norm is close to the minimal norm, of degree less than 40. We then use the irreducible factors of such a polynomial as polynomials $Q_{j}$ in the auxiliary function. So, to deal with the general situation, i.e., for varying $t$, we first take an initial value of $t$ ( say $t_{0}=1$ ) and compute a polynomial $H$ of small degree (if possible less than 5). We define $Q_{1}$ as an irreducible factor of $H$, and we take the best value for $e_{1}$ to get the best auxiliary function $f_{1}$. We deduce from this the value of $t=t_{1}$, and we compute a new polynomial $H$ to get a new irreducible factor $Q_{2}$. Then we optimize the function $f_{2}$ with the two factors $Q_{1}$ 
and $Q_{2}$. We continue this process until the degree of $H$ becomes too large. For the optimization of the auxiliary function, we use a refinement of the semi-infinite linear programming method introduced into number theory by Smyth [SM84].

\section{Bounds FOR $s_{k}$ FOR POlynomials With SMALl MahleR MEASURE}

In the rest of the paper we assume that the polynomials $P$ satisfy the conditions of Section 2, where $S$ is the set of polynomials listed in Table 3 (at the end of this section).

Throughout this section we will use an explicit auxiliary function $f$ of the following type:

$$
f(z)=\operatorname{Re}(z)-\sum_{1 \leq j \leq J} e_{j} \log \left|Q_{j}(z)\right| .
$$

Here $z$ is a complex number, the $e_{j}$ are positive real numbers and the $Q_{j}$ are those given in Table 3 . Note that the numbers $e_{j}$ are always chosen to get the best auxiliary function. For the degree 34 we use 1111 different auxiliary functions.

For $a \geq 0$ we define the function $g$ as

$$
g(a)=\min _{z \in \mathcal{E}_{a}} f(z) .
$$

We need the following lemma.

Lemma 1. 1. Let $n \geq 1$ be an integer and let $a_{1}, \ldots, a_{n}, b$ be $n+1$ real numbers satisfying the conditions $b>0, a_{i} \in[0, b]$ for $1 \leq i \leq n$ and $\sum_{1 \leq i \leq n} a_{i} \leq b$. Let $h$ be a linear decreasing real function on $[0, b]$. Then

$$
\sum_{1 \leq i \leq n} h\left(a_{i}\right) \geq(n-1) h(0)+h(b)
$$

2. We now suppose that $n>1$ and all the numbers $a_{i} \in[0, b / 2]$. Then

$$
\sum_{1 \leq i \leq n} h\left(a_{i}\right) \geq(n-2) h(0)+2 h(b / 2) .
$$

Proof. 1. We have

$$
\sum_{1 \leq i \leq n} h\left(a_{i}\right) \geq \sum_{1 \leq i \leq n-1} h\left(a_{i}\right)+h\left(b-\sum_{1 \leq i \leq n-1} a_{i}\right),
$$

and the right term is constant and equal to $(n-1) h(0)+h(b)$.

2. If $\sum_{1 \leq i \leq n-1} a_{i}$ is not greater than $b / 2$, then we apply (5.3) to this partial sum (with $b$ replaced by $b / 2$ ) and take the trivial lower bound $h(b / 2)$ for the last term. Otherwise we proceed as in case 1 and get, as in (5.5), a constant term which is equal to the right term of (5.4).

a. A lower bound for $s_{1}$ in the second case.

We first examine the second case which is the simpler one, i.e., when the roots $\boldsymbol{\alpha}_{i}$ satisfy $1 \leq\left|\boldsymbol{\alpha}_{i}\right| \leq M^{1 / 2}$ for $1 \leq i \leq d$. Then all the roots $\gamma_{i}$ of $Q$ belong to the ellipse $\mathcal{E}_{1 / 2}$. Since $\gamma_{i} \in \mathcal{E}_{a_{i}}$ with $a_{i}=\log \left|\boldsymbol{\alpha}_{i}\right| / \log M$, we have

$$
\sum_{1 \leq i \leq d} f\left(\gamma_{i}\right) \geq \sum_{1 \leq i \leq d} g\left(a_{i}\right)
$$


and, since $Q$ does not divide any $Q_{j}$,

$$
s_{1} \geq \sum_{1 \leq i \leq d} g\left(a_{i}\right) .
$$

If we have a linear decreasing function $h$ such that, for all $x \in[0,1 / 2], h(x) \leq$ $g(x)$ (we write now $h \leq g$ ), then, using case 2 of Lemma 1, we get from (5.6)

$$
s_{1} \geq(d-2) h(0)+2 h(1 / 2) .
$$

For a fixed $\varepsilon$ (say $\varepsilon=1 / 10$ ), we want to find a function $h \leq g$ such that

$$
(d-2) h(0)+2 h(1 / 2) \geq(d-2) g(0)+2 g(1 / 2)-\varepsilon .
$$

Now we explain how to choose a suitable function $h$ in a special case. For $2 d=34$, let $f$ be the auxiliary function which is used to get a lower bound for $s_{1}$. The polynomials $Q_{j}$ are the following 11 polynomials given in Table 3 :

$$
Q_{1}, Q_{2}, Q_{4}, Q_{8}, Q_{9}, Q_{11}, Q_{13}, Q_{14}, Q_{17}, Q_{20}, Q_{21},
$$

and the coefficients $e_{j}$ are, respectively, equal to

$0.14768307,0.49117397,0.56247516,0.03015563,0.22586214,0.05159352$,

$$
0.15415127,0.02119227,0.05373559,0.04126272,0.04828564 .
$$

We have

$$
g(0)=-0.20529481 \cdots, \quad g(1 / 2)=-0.67503341 \cdots,
$$

and

$$
(d-2) g(0)+2 g(1 / 2)=-4.42948908 \cdots .
$$

Put

$$
\begin{gathered}
\varepsilon_{0}=\frac{\varepsilon}{2(d-2)}, \quad \varepsilon_{1}=\frac{\varepsilon}{4}, \\
m_{0}=g(0)-\varepsilon_{0}, \quad m_{1}=g(1 / 2)-\varepsilon_{1} .
\end{gathered}
$$

The function $h$ is defined by $h(0)=m_{0}$ and $h(1 / 2)=m_{1}$. We find a staircase function $s$ such that $h \leq s \leq g$. The sequence $0<x_{t}<\cdots<x_{2}<x_{1}=1 / 2$ of points where $s$ is not continuous is defined by induction as follows. We begin at $x_{1}=1 / 2$, where $s\left(x_{1}\right)=g\left(x_{1}\right)$. We define $x_{2}$, which is $<x_{1}$, by $h\left(x_{2}\right)=g\left(x_{1}\right)$. Since $g$ and $h$ are decreasing we have, for any $x \in\left(x_{2}, x_{1}\right), h(x)<s(x)=g\left(x_{1}\right)<g(x)$, and we continue until $g\left(x_{t}\right) \geq m_{0}$. The last stair is $\left(0, x_{t}\right)$, where $s$ has the value $g\left(x_{t}\right)$. In our special case $t=13$ and $(d-2) h(0)+2 h(1 / 2) \geq-4.52948908$, so $s_{1} \geq-4$.

Now we have

$$
s_{1} \geq(d-2) \min _{x \in \mathcal{E}_{0}} f(x)+2 \min _{z \in \mathcal{E}_{1 / 2}} f(z)-\varepsilon,
$$

and we proceed as in Section 3. We replace the numbers $e_{j}$ by rational numbers, and we take the exponential. Then the right side of inequality (5.7) becomes

$$
\left(\sup _{x \in \mathcal{E}_{0}}|H(x)|^{t / h} e^{-x}\right)^{d-2}\left(\sup _{z \in \mathcal{E}_{1 / 2}}|H(z)|^{t / h} e^{-\operatorname{Re}(z)}\right)^{2},
$$

where $H \in \mathbb{Z}[X]$. Since, for $d$ large and $M$ sufficiently close to 1 , the first factor in (5.8) is the larger one, we apply Wu's algorithm to $\sup _{x \in \mathcal{E}_{0}}|H(x)|^{t / h} e^{-x}$ to get the polynomials $Q_{j}$. Once we have obtained a list of polynomials $Q_{j}$, it remains to get the best real numbers $e_{j}$. 
b. The semi-infinite linear programming method.

Since $f$ is a harmonic function, the minimum of $f(z)$ in the ellipse $\mathcal{E}_{1 / 2}$ is taken on its boundary $\mathcal{B}\left(\mathcal{E}_{1 / 2}\right)$. It is clear that, if in (5.7) we take the minimum on a finite set of points of $[-2,2] \cup \mathcal{B}\left(\mathcal{E}_{1 / 2}\right)$, we obtain a value which is greater than or equal to the right side of (5.7). Moreover, if we replace $\operatorname{Re}(z)$ by $e_{0} \operatorname{Re}(z)$ with the condition $e_{0}=1$, we may find the best numbers $e_{j}$ by linear programming. We define by induction a sequence of finite sets $X_{n}, n \geq 0$, as $X_{n}=Y_{n} \cup Z_{n}$ with $Y_{n} \subset \mathcal{E}_{0}$ and $Z_{n} \subset \mathcal{B}\left(\mathcal{E}_{1 / 2}\right)$. We start with an arbitrary set of points $X_{0}$ of cardinal greater than $J$. At each step $n \geq 0$ we compute the best numbers $e_{j}$ by linear programming with the set of points $X_{n}$. We get a function $f_{n}$. This gives a minimum

$$
\mu_{n}=(d-2) \min _{x \in Y_{n}} f_{n}(x)+2 \min _{z \in Z_{n}} f_{n}(z) .
$$

This quantity is greater than

$$
m_{n}=(d-2) \min _{x \in \mathcal{E}_{0}} f_{n}(x)+2 \min _{z \in \mathcal{B}\left(\mathcal{E}_{1 / 2}\right)} f_{n}(z) .
$$

Let $m_{n, 0}=\min _{x \in \mathcal{E}_{0}} f_{n}(x)$ and $m_{n, 1}=\min _{z \in \mathcal{B}\left(\mathcal{E}_{1 / 2}\right)} f_{n}(z)$. We add to the set $Y_{n}$ (resp. $Z_{n}$ ) the points of $\mathcal{E}_{0}$ (resp. $\mathcal{B}\left(\mathcal{E}_{1 / 2}\right)$ ), where $f_{n}$ has a local minimum which is smaller than $m_{n, 0}+c_{n, 0}$ (resp. $\left.m_{n, 1}+c_{n, 1}\right) \cdot c_{n, 0}$ and $c_{n, 1}$ are decreasing sequences of positive numbers tending to 0 when $n$ is increasing. These sequences are chosen such that the set $X_{n}$ does not increase too quickly. After a small number of steps, the integer parts of $\mu_{n}$ and $m_{n}$ are equal, and we stop. This gives a lower bound for $s_{1}$.

c. The bounds for $s_{k}$ in the second case.

To obtain an upper bound for $s_{k}$ it is sufficient to replace $M$ by $M^{k}$ and to replace $\boldsymbol{\alpha}_{i}$ by $\boldsymbol{\alpha}_{i}^{k}$. Since the numbers $-\boldsymbol{\alpha}_{i}$ satisfy the same conditions as $\boldsymbol{\alpha}_{i},-s_{k}$ has the same upper bound as $s_{k}$.

d. A numerical example in the second case.

The polynomial $P_{3}=X^{38}+X^{37}-X^{29}-X^{19}-X^{9}+X+1$ has a Mahler measure equal to $1.308128 \cdots<1.31$, and it has only two complex conjugate roots of modulus $>1$. The classical bound for $s_{k}$, when we are in the second case, is $\left|s_{k}\right| \leq 2 M^{k / 2}+2 d-4+2 M^{-k / 2}$. We give in Table 2 , for some values of $k$, the value of $s_{k}$ for $P_{3}$, the bound that we obtain $(\max )$ and the value of the classical bound (MAX).

TABLE 2 .

\begin{tabular}{|c|c|c|c|c|c|c|c|c|c|c|}
\hline$k$ & 1 & 2 & 3 & 4 & 10 & 19 & 28 & 38 & 39 & 40 \\
\hline$s_{k}$ & -1 & 1 & -1 & 1 & -9 & 18 & 99 & -246 & 324 & -409 \\
\hline $\max$ & 4 & 6 & 7 & 8 & 17 & 41 & 106 & 360 & 409 & 465 \\
\hline MAX & 38 & 38 & 38 & 38 & 42 & 60 & 121 & 372 & 421 & 477 \\
\hline
\end{tabular}

e. Bounds for $s_{k}$ in the first case.

Now we examine the case where $Q$ has a positive real root $\boldsymbol{\alpha}_{1}>M^{1 / 2}$ so $\boldsymbol{\alpha}_{1}$ belongs to one of the 6 intervals $\left[t_{1}(j), t_{2}(j)\right]$ defined in Section 2. Then all the other roots $\gamma_{2}, \ldots, \gamma_{d}$ of $Q$ belong to the ellipse $\mathcal{E}_{1 / 2-(j-1) / 12}$. We get

$$
\sum_{1 \leq i \leq d} f\left(\gamma_{i}\right) \geq \sum_{2 \leq i \leq d} g\left(a_{i}\right)+\min _{t_{1} \leq x \leq t_{2}} f(x),
$$

where $a_{i}=\log \left|\boldsymbol{\alpha}_{i}\right| / \log M$. 
As in item a above we get a suitable linear decreasing function $h$ defined on the interval $[0,1 / 2-(j-1) / 12]$ such that $g(a) \geq h(a)$ for $a \in[0,1 / 2-(j-1) / 12]$.

We use the case 1 of Lemma 1 and get from (5.9)

$$
s_{1} \geq(d-2) h(0)+h(1 / 2-(j-1) / 12)+\min _{t_{1} \leq x \leq t_{2}} f(x)
$$

and, as in item a,

$$
s_{1} \geq(d-2) \min _{x \in \mathcal{E}_{0}} f(x)+\min _{z \in \mathcal{E}_{1 / 2-(j-1) / 12}} f(z)+\min _{t_{1} \leq x \leq t_{2}} f(x)-\varepsilon .
$$

We use the list of polynomials $Q_{j}$ of Table 3 , and for the semi-infinite linear programming we get a sequence of finite sets of points in $[-2,2] \cup \mathcal{B}\left(\mathcal{E}_{1 / 2-(j-1) / 12}\right) \cup$ $\left[t_{1}, t_{2}\right]$.

For the upper bound of $s_{1}$ we use the numbers $-\boldsymbol{\alpha}_{i}$ and, similar to (5.11), we get

$$
-s_{1} \geq(d-2) \min _{x \in \mathcal{E}_{0}} f(x)+\min _{z \in \mathcal{E}_{1 / 2-(j-1) / 12}} f(z)+\min _{-t_{2} \leq x \leq-t_{1}} f(x)-\varepsilon .
$$

For $k>1$ we replace $M$ by $M^{k}$ and we obtain two inequalities of the same kind as (5.11) and (5.12).

Table 3 gives the list of polynomials $Q_{j}$ of the set $S$ which are used in the auxiliary functions of Sections 5 and $6 . d=\operatorname{deg} Q_{j}$ and the coefficients of $Q_{j}$ are

\begin{tabular}{|c|c|c|c|c|c|c|c|}
\hline $\bar{Q}$ & $d$ & \multicolumn{6}{|c|}{ Coefficients of $Q$} \\
\hline$Q_{1}$ & 1 & 1 & 0 & & & & \\
\hline$Q_{2}$ & 1 & 1 & 1 & & & & \\
\hline$Q_{3}$ & 1 & 1 & -1 & & & & \\
\hline$Q_{4}$ & 1 & 1 & 2 & & & & \\
\hline$Q_{5}$ & 1 & 1 & -2 & & & & \\
\hline$Q_{6}$ & 1 & 2 & 3 & & & & \\
\hline$Q_{7}$ & 1 & 3 & 5 & & & & \\
\hline$Q_{8}$ & 2 & 1 & 0 & -2 & & & \\
\hline$Q_{9}$ & 2 & 1 & 1 & -1 & & & \\
\hline$Q_{10}$ & 2 & 1 & -1 & -1 & & & \\
\hline$Q_{11}$ & 2 & 1 & 0 & -3 & & & \\
\hline$Q_{12}$ & 2 & 2 & 4 & 1 & & & \\
\hline$Q_{13}$ & 3 & 1 & 1 & -2 & -1 & & \\
\hline$Q_{14}$ & 3 & 1 & 0 & -3 & 1 & & \\
\hline$Q_{15}$ & 3 & 3 & 9 & 6 & -1 & & \\
\hline$Q_{16}$ & 3 & 2 & -3 & -2 & 1 & & \\
\hline$Q_{17}$ & 4 & 1 & 1 & -4 & -4 & 1 & \\
\hline$Q_{18}$ & 4 & 1 & 1 & -5 & -7 & -1 & \\
\hline$Q_{19}$ & 4 & 4 & 15 & 16 & 3 & -1 & \\
\hline$Q_{20}$ & 5 & 1 & 1 & -4 & -3 & 3 & 1 \\
\hline$Q_{21}$ & 5 & 1 & 1 & -5 & -5 & 4 & 3 \\
\hline
\end{tabular}
written from degree $d$ to 0 .

\section{TABLE 3.}




\section{A REFINEMENT OF THE METHOD FOR THE BOUNDS FOR $s_{k}$}

In order to get a smaller set $E_{1}$ we need more information on the numbers $s_{k}$. In this section we consider only the second case, because it gives the most important contribution to $E_{1}$. Our heuristic idea is that " $s_{k}$ and $s_{2 k}$ cannot be too close to their bounds simultaneously." Here the classical inequality BO80] may be written as

$$
s_{2 k} \geq \frac{1}{d}\left(s_{k}\right)^{2}-2 M^{k}-(2 d-4)-2 M^{-k} .
$$

We consider in this section an explicit auxiliary function $f$ of the following type:

$$
f(z)=\operatorname{Re}\left(z^{2}-2\right)+e_{0} \operatorname{Re}(z)-\sum_{1 \leq j \leq J} e_{j} \log \left|Q_{j}(z)\right|
$$

with the same conditions as before for the numbers $e_{j}$ for $0 \leq j \leq J$ and for the polynomials $Q_{j}$ which belong to the set $S$ defined in Section 5 . Doing the summation on the values of $f$ at the numbers $\gamma_{i}$ we get

$$
\sum_{1 \leq i \leq d} f\left(\gamma_{i}\right) \geq(d-2) \min _{x \in \mathcal{E}_{0}} f(x)+2 \min _{z \in \mathcal{E}_{1 / 2}} f(z)-\varepsilon=m-\varepsilon .
$$

Since $\gamma_{i, 2}=\boldsymbol{\alpha}_{i}^{2}+1 / \boldsymbol{\alpha}_{i}^{2}=\left(\boldsymbol{\alpha}_{i}+1 / \boldsymbol{\alpha}_{i}\right)^{2}-2=\gamma_{i}^{2}-2$ we get $s_{2}+e_{0} s_{1} \geq m-\varepsilon$. If we assume that $s_{1}$ has the value $\boldsymbol{\sigma}$, then

$$
s_{2} \geq m-e_{0} \boldsymbol{\sigma}-\varepsilon
$$

So, we want to get the largest value for the right-side of (6.3) which is linear in the numbers $e_{j}$. We maximize $m-e_{0} \boldsymbol{\sigma}$. Therefore we get a lower bound for $s_{2}$ depending on the value $\boldsymbol{\sigma}$ of $s_{1}$. When the value $\boldsymbol{\sigma}$ increases from the lower bound of $s_{1}$ that has been computed in Section 5 , say $-B_{1}$, the bound for $s_{2}$ decreases. We stop when this lower bound is less than $-B_{2}$, which is the lower bound of $s_{2}$ that has been computed in Section 5. Of course we may replace the numbers $\boldsymbol{\alpha}_{i}$ by the numbers $-\boldsymbol{\alpha}_{i}$. If we replace $+e_{0} \operatorname{Re}(z)$ by $-e_{0} \operatorname{Re}(z)$ in $(6.2)$, we get the same lower bound for $s_{2}$ when $s_{1}$ takes the value $-\boldsymbol{\sigma}$. We may also replace $\operatorname{Re}\left(z^{2}-2\right)$ by $-\operatorname{Re}\left(z^{2}-2\right)$ in $(6.2)$. Then we get an upper bound for $s_{2}$ depending on the value of $\left|s_{1}\right|$. Then we replace the numbers $\boldsymbol{\alpha}_{i}$ by the numbers $\pm \boldsymbol{\alpha}_{i}^{k}$ and $M$ by $M^{k}$ for $1 \leq k \leq 20$, and we get lower and upper bounds for $s_{2 k}$ depending on the value of $\left|s_{k}\right|$.

\section{Decreasing the set $E_{1}$}

Since we know all the coefficients of $P$, it is possible to compute all the numbers $s_{k}$ for $d+1 \leq k \leq 40$. Now, as D. Boyd in [BO85], we eliminate any polynomial $P$ of $E_{1}$ for which $s_{k}$ is not inside its bounds (those computed in Section 5) for any $k \leq 40$, and for even $k=2 l$ we verify that, for this value for $s_{l}, s_{2 l}$ is between the bounds that have been computed in Section 6 .

In the first case, since $P$ has only one root $\boldsymbol{\alpha}_{1}$ for $x>t_{1}=t_{1}(j)$, the function $P(x)$ is convex in this range. Therefore the line joining the points $\left(t_{1}, P\left(t_{1}\right)\right)$ and $\left(t_{2}, P\left(t_{2}\right)\right)$ cuts the real axis at a value $t_{3}<\boldsymbol{\alpha}_{1}$. Then all the other roots of $P$ are in the disk of radius $M_{1}=M / t_{3}$.

In the second case all the roots of $P$ lie in the disk of radius $M^{1 / 2}$. In the two cases we compute the number of roots of $P$ which lie outside a sequence of disks of decreasing radius by the Schur-Cohn algorithm $[\mathrm{MA}$. This will give a lower bound 
of $M(P)$ which has to be $<M$. After the elimination of the polynomials which have cyclotomic factors of small degree, i.e., the degree of the associated polynomial is $\leq 3$, we get the new set $E_{2}$ of polynomials $P$. Then we use GP-Pari [PARI] to get the set $E_{0}$ of all the irreducible primitive polynomials with Mahler measure less than $M$.

\section{The Final COMputation}

We first eliminate the polynomials which have a factor associated to one of the 13 cyclotomic polynomials of degrees 8 to 12 . Then we use a modified Graeffe algorithm. For this purpose we need

Lemma 2. Let $P$ be a nonzero polynomial in $\mathbb{C}[X]$ of degree $n$. Let $L(P)$ be the length of $P$; that is, the sum of the modulus of the coefficients of $P$. Then we have

$$
M(P) \leq L(P) \leq 2^{n} M(P) .
$$

We now suppose that $P$ is a reciprocal polynomial of degree $2 d$ and that $Q$ is the polynomial of degree $d$ associated to $P$. Then we have

$$
\omega^{-d} M(P) \leq L(Q) \leq 3^{d} M(P)
$$

where $\omega$ is the golden ratio $\frac{1+\sqrt{5}}{2}=1.618033 \cdots$.

Proof. Let $\boldsymbol{\alpha}_{1}, \ldots, \boldsymbol{\alpha}_{d}$ be $d$ roots of $P$ of modulus $\geq 1$ and $1 / \boldsymbol{\alpha}_{1}, \ldots, 1 / \boldsymbol{\alpha}_{d}$ the last $d$ other roots. We have

$$
P(X)=c_{0} \prod_{1 \leq i \leq 2 d}\left(X-\boldsymbol{\alpha}_{i}\right) \quad \text { and } \quad Q(X)=c_{0} \prod_{1 \leq i \leq d}\left(X-\left(\boldsymbol{\alpha}_{i}+1 / \boldsymbol{\alpha}_{i}\right)\right) .
$$

The inequalities in (8.1) are well known and are used in Graeffe's algorithm (see BO80]). It is plain that

$$
L(Q) \leq\left|c_{0}\right| \prod_{1 \leq i \leq d}\left(1+\left|\boldsymbol{\alpha}_{i}\right|+1 /\left|\boldsymbol{\alpha}_{i}\right|\right)
$$

and that for any $1 \leq i \leq d$

$$
1+\left|\boldsymbol{\alpha}_{i}\right|+1 /\left|\boldsymbol{\alpha}_{i}\right| \leq 3 \max \left(1,\left|\boldsymbol{\alpha}_{i}\right|\right) .
$$

So we get the right-hand inequality in (8.2). By (8.1) we have $M(Q) \leq L(Q)$ and

$$
M(Q)=\left|c_{0}\right| \prod_{1 \leq i \leq d} \max \left(1,\left|\boldsymbol{\alpha}_{i}+1 / \boldsymbol{\alpha}_{i}\right|\right)
$$

If $\left|\boldsymbol{\alpha}_{i}\right|>\omega$, then

$$
\left|\boldsymbol{\alpha}_{i}+1 / \boldsymbol{\alpha}_{i}\right| \geq\left|\boldsymbol{\alpha}_{i}\right|\left(1-1 /\left|\boldsymbol{\alpha}_{i}\right|^{2}\right) \geq\left|\boldsymbol{\alpha}_{i}\right|\left(1-1 / \omega^{2}\right)=\left|\boldsymbol{\alpha}_{i}\right| / \omega
$$

If $1 \leq\left|\boldsymbol{\alpha}_{i}\right| \leq \omega$, then $1 \geq\left|\boldsymbol{\alpha}_{i}\right| / \omega$. Since $M(P)=\left|c_{0}\right| \prod_{1 \leq i \leq d}\left|\boldsymbol{\alpha}_{i}\right|$, this proves the left-hand inequality of (8.2).

Remark. The inequalities in (8.1) and the right-hand one in (8.2) are the best possible. For (8.1) take the polynomials $X^{n}$ and $(X+1)^{n}$ for the left- and righthand side, respectively. For the right-hand side of $(8.2)$ take $P=(X+1)^{2 d}$, then $M(P)=1$ and $Q=(X+2)^{d}$, which gives $L(Q)=3^{d}$. The constant $\omega^{-d}$ in the left-hand side of (8.2) is perhaps not the best one, but it cannot be replaced by 1 . If we take $P=\left(X^{4}+3 X^{2}+1\right)^{n}$, then $d=2 n, M(P)=\omega^{d}$ and $Q=\left(X^{2}+1\right)^{n}$, so $L(Q)=2^{n}=(\sqrt{2})^{d}, M(P)=\omega^{d} M(Q)$ and $M(P)=L(Q)(1.144 \cdots)^{d}$. 
The inequalities in (8.1) are the basic tools for the Graeffe algorithm. If $P_{1}$ is the polynomial whose roots are the squares of the roots of $P$, then $L\left(P_{1}\right) \leq$ $2^{\operatorname{deg}(P)} M\left(P_{1}\right)=2^{\operatorname{deg}(P)} M(P)^{2}$. Iterating this process, we get a sequence of polynomials $P_{m}$ for $m>0$, and we have

$$
M(P)=M\left(P_{m}\right)^{2^{-m}} \leq L\left(P_{m}\right)^{2^{-m}} \leq 2^{\operatorname{deg}(P) / 2^{m}} M(P) .
$$

This sequence of inequalities shows that, if $M(P)>M$, after a finite number of root-squaring we will have $L\left(P_{m}\right)>2^{\operatorname{deg}(P)} M^{2^{m}}$. Since the constants in (8.2) are smaller than in (8.1) we will use the inequalities (8.2) for a modified Graeffe algorithm. We have

$$
X-\boldsymbol{\alpha}_{i}^{2}-1 / \boldsymbol{\alpha}_{i}^{2}=X+2-\left(\boldsymbol{\alpha}_{i}+1 / \boldsymbol{\alpha}_{i}\right)^{2},
$$

therefore the polynomial $Q_{1}$ associated to $P_{1}$ may be defined by the following formulae. Let $g$ and $h$ be the two polynomials such that

$$
Q(X)=g\left(X^{2}\right)+X h\left(X^{2}\right) ;
$$

then

$$
Q_{1}(X)=g(X+2)^{2}-(X+2) h(X+2)^{2} .
$$

Let us give an example. Let $P$ be the polynomial

$$
\begin{gathered}
X^{40}+X^{39}+X^{34}+X^{33}+X^{28}-X^{26}-X^{23}-X^{21}-3 X^{20} \\
-X^{19}-X^{17}-X^{14}+X^{12}+X^{7}+X^{6}+X+1 .
\end{gathered}
$$

Then $M(P)=1.395928 \cdots>1.31$ and

$$
L\left(P_{8}\right)=5.29 \cdots 10^{39}<2^{40} 1.31^{2^{8}}=1.15 \cdots 10^{42},
$$

but

$$
L\left(Q_{8}\right)=7.44 \cdots 10^{39}>3^{20} 1.31^{2^{8}}=3.66 \cdots 10^{39} .
$$

We test the polynomials $Q_{m}$ until $m=9$, and we keep only those which survive all the tests. Then, for the polynomials $Q$ which are irreducible, we compute their roots and the measure of $P$.

\section{REFERENCES}

[B] P. Borwein, Computational Excursions in Analysis and Number Theory, CMS Books in Mathematics, Springer (2002). MR.1912495 (2003m:11045)

[BE] P. Borwein and T. Erdélyi, The integer Chebyshev problem, Math. Comp. 65 (1996), 661-681. MR 1333305 (96g:11077)

[BO80] D.W. Boyd, Reciprocal polynomials having small measure, Math. Comp. 35 (1980), 13611377. MR0583514 (82a:30005)

[BO85] D.W.Boyd, The maximal modulus of an algebraic integer, Math. Comp. 45 (1985), 243-249. MR0790657 (87c:11097)

[BO89] D.W. Boyd, Reciprocal polynomials having small measure II, Math. Comp. 53 (1989), 355-357; S1-S5. MR0968149 (89m:30013)

[DHL] D.H. Lehmer, Factorization of certain cyclotomic functions, Ann. of Math. 34 (1933), 461-479. MR1503118

[FR] V.Flammang and G. Rhin, Algebraic integers whose conjugates all lie in an ellipse, Math. Comp. 74 (2005), 2007-2015.

[FRS] V.Flammang, G. Rhin, and C.J.Smyth, The integer transfinite diameter of intervals and totally real algebraic integers, J. Théor. Nombres Bordeaux 9 (1997), 137-168. MR:1469665 (98g:11119)

[FGR] V. Flammang, M. Grandcolas, and G. Rhin, Small Salem numbers, Proceedings of the International Conference on Number Theory, Zakopane, 1997, Number Theory in Progress, Walter de Gruyter (1999), 165-168. MR1689505 (2000e:11132) 
[MA] M. Marden, Geometry of polynomials, Amer. Math. Soc., Providence, Rhode Island (1966). MR0225972 (37:1562)

[MO] M.J. Mossinghoff, Polynomials with small Mahler measure, Math. Comp. 67 (1998), 1697-1705; S11-S14. MR1604391 (99a:11119)

[PARI] C. Batut, K. Belabas, D. Bernardi, H. Cohen, and M. Olivier, GP-Pari version 2.0.12, 1998.

[PR] I. Pritsker, Small polynomials with integer coefficients (preprint).

[RSE] G. Rhin and J.-M. Sac-Épée, New methods providing high degree polynomials with small Mahler measure, Exp. Maths. 12 (2003), 457-461. MR2043995 (2005a:11165)

[SM71] C.J.Smyth, On the product of the conjugates outside the unit circle of an algebraic integer, Bull. London Math. Soc. 3 (1971), 169-175. MR0289451 (44:6641)

[SM84] C.J.Smyth, The mean values of totally real algebraic integers, Math. Comp. 42 (1984), 663-681. MR0736460 (86e:11115)

[SM03] C.J. Smyth, Private communication, 2003.

[V] P. Voutier, An effective lower bound for the height of algebraic numbers, Acta Arith. 74 (1996), 81-95. MR.1367580(96j:11098)

[WE] http://www.math.univ-metz.fr/ ${ }^{\sim}$ rhin.

[WM] http://www.cecm.sfu.ca/ ${ }^{\sim m j m / L e h m e r / . ~}$

[WU] Q. Wu, On the linear independence measure of logarithms of rational numbers, Math. Comp. 72 (2002), 901-911. MR.1954974 (2003m:11111)

UMR CNRS 7122, Département de Mathématiques, UFR MiM, Université de Metz, Ile du Saulcy, 57045 Metz Cedex 01, France

E-mail address: flammang@poncelet.univ-metz.fr

UMR CNRS 7122, Département de Mathématiques, UFR Mim, Université de Metz, Ile du Saulcy, 57045 Metz Cedex 01, France

E-mail address: rhin@poncelet.univ-metz.fr

UMR CNRS 7122, Département de Mathématiques, UFR Mim, Université de Metz, Ile du Saulcy, 57045 Metz Cedex 01, France

E-mail address: jmse@poncelet.univ-metz.fr 